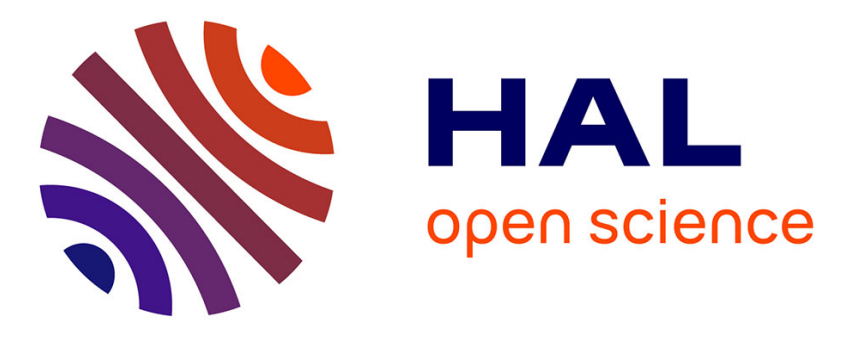

\title{
Self-organized arrays of dislocations in thin smectic liquid crystal films
}

Delphine Coursault, Bruno Zappone, Alessandro Coati, Athmane Boulaoued, Laurent Pelliser, Denis Limagne, Nathalie Boudet, Bicher Haj Ibrahim, Antonello de Martino, Michel Alba, et al.

\section{To cite this version:}

Delphine Coursault, Bruno Zappone, Alessandro Coati, Athmane Boulaoued, Laurent Pelliser, et al.. Self-organized arrays of dislocations in thin smectic liquid crystal films. Soft Matter, 2016, 12 (3), pp.678-688. 10.1039/C5SM02241J . hal-01230908

\section{HAL Id: hal-01230908 https://hal.sorbonne-universite.fr/hal-01230908}

Submitted on 19 Nov 2015

HAL is a multi-disciplinary open access archive for the deposit and dissemination of scientific research documents, whether they are published or not. The documents may come from teaching and research institutions in France or abroad, or from public or private research centers.
L'archive ouverte pluridisciplinaire HAL, est destinée au dépôt et à la diffusion de documents scientifiques de niveau recherche, publiés ou non, émanant des établissements d'enseignement et de recherche français ou étrangers, des laboratoires publics ou privés. 


\section{Self-organized arrays of dislocations in thin smectic liquid crystal films}

Delphine Coursault, ${ }^{a}$ Bruno Zappone,${ }^{b}$ Alessandro Coati,${ }^{c}$ Athmane Boulaoued, ${ }^{a d e}$ Laurent Pelliser, ${ }^{a}$ Denis Limagne, ${ }^{a}$ Nathalie Boudet, ${ }^{f}$ Bicher Haj Ibrahim, ${ }^{g}$ Antonello de Martino, ${ }^{g}$ Michel Alba, ${ }^{h}$ Michel Goldmann, ${ }^{a i}$ Yves Garreau, ${ }^{b j}$ Bruno Gallas, ${ }^{a}$ Emmanuelle Lacaze ${ }^{* a}$

Combining optical microscopy, synchrotron X-ray diffraction and ellipsometry, we studied the internal structure of linear defect domains (oily streaks) in films of smectic liquid crystal 8CB with thickness $100-300 \mathrm{~nm}$ confined between air and a rubbed PVA polymer substrate which impose hybrid anchoring conditions (normal and unidirectional planar, respectively). We show how the presence or absence of dislocations control the structure of highly deformed thin smectic films. Each domain contains smectic layers curved in the shape of flattened hemicylinders to satisfy both anchoring conditions, together with grain boundaries whose size and shape are controlled by the presence of dislocation lines. A flat grain boundary normal to the interface connects neighboring hemicylinders, while a rotating grain boundary (RGB) is located near the axis of curvature of the cylinders. The RGB shape appears such that dislocation lines are concentrated at its summit close to the air interface. The smectic layers reach the polymer substrate via a transition region where the smectic layer orientation satisfies the planar anchoring condition over the entire polymer substrate and whose thickness does not depend on the one of the film. The strength of the planar anchoring appears to be high, larger than $10^{-2} \mathrm{~J} / \mathrm{m}^{2}$, compensating for the high energy cost of creating an additional 2D defect between an horizontal smectic layer and perpendicular ones. This 2D defect may be melted, in order to avoid the creation of a transition region structure composed of a large number of dislocations. As a result, linear defect domains can be considered as arrays of oriented defects, straight dislocations of various Burger vectors, whose location is now known and 2D nematic defects. The possibility of easy variation between the present structure with a moderate amount of dislocations and a structure with a large number of dislocations is also demonstrated.

\section{Introduction}

Liquid crystal phases, smectic liquid crystal phase in particular, can host a variety of topological defects. The physics of topological defects is related to a large field of research? including mathematics ${ }^{?}$, cosmology ${ }^{?}$ or superconductor vortices? ${ }^{?}$. Smectic topological defects have been extensively

${ }^{a}$ CNRS UMR 7588, Sorbonne Universités, UPMC Univ Paris 06, Institut des NanoSciences de Paris (INSP), 4 place Jussieu, 75005 Paris, France; E-mail: emmanuelle.lacaze@insp.jussieu.fr

${ }^{b}$ Consiglio Nazionale delle Recerche, CNR-NANOTEC, UOS LICRYLCosenza, 87036 Rende (CS), Italy

'Synchrotron Soleil, BP 48, L'Orme des Merisiers, 91192 Gif sur Yvette Cedex, France

${ }^{d}$ Laboratoire des Matériaux, Surfaces et Procédés pour la Catalyse, UMR 7515 CNRS, Université de Strasbourg, 25 rue Becquerel, F-67087 Strasbourg Cedex 2, France

${ }^{e}$ Laboratoire POMAM, UMR 7177 CNRS, Université de Strasbourg, 1 rue Blaise Pascal, BP 296 R8, F-67008 Strasbourg Cedex, France

${ }^{f}$ Institut NEEL/CNRS-UJF, 25 rue des Martyrs, Grenoble, France

${ }^{g}$ Laboratoire de Physique des Interfaces et Couches Minces (LPICM), CNRS UMR 7647, Ecole polytechnique, 91128, Palaiseau, France

${ }^{h}$ Laboratoire Léon Brillouin UMR12 CNRS-CEA, CEA Saclay, 91191 Gif sur Yvette Cedex, France

${ }^{i}$ UFR Biomédicale des Saints-Pères, Université Paris Descartes, 45 rue des St Pères, 75006 Paris, France

${ }^{j}$ Sorbonne-Paris-Cité, Matériaux et Phénomenes Quantiques (MPQ), Université Paris Diderot, UMR 7162 CNRS, Bâtiment Condorcet, Case 7021, 75205 Paris Cedex 13, France

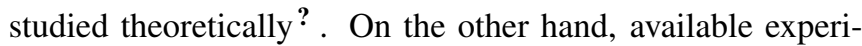
mental data on the exact structure of the defects are still scarce, especially when the nanoscale is concerned. The necessity of comprehensive data on the structure of smectic defects becomes even more important nowadays, while a number of new smectic systems are under development. They include smectic droplets ? ?, smectic shells ? ??, but also arrays of focal conics created by curved interfaces? ? ?, all being expected to present a number of topological defects. New periodic structures based on selective evaporation of liquid crystal molecules in presence of focal conics are created? ${ }^{\text {. More- }}$ over, it has been shown that liquid crystal topological defects constitute efficient traps for nanoparticles. When nanoparticles are trapped within the defect cores, the energy of the cores is released, leading to a stabilization of the composite system? ? ? . The phenomenon however depends on the nanoparticle size ${ }^{\text {? }}$. If the nanoparticle size matches the one of the defect core, in presence of oriented linear topological defects, formation of linear assemblies of nanoparticles may occur ${ }^{?}$ ? . For the creation of highly anisotropic assemblies (with anisotropic optical properties? ? ), the density of oriented linear topological defects has to be high enough with respect to the nanoparticles density. Otherwise, nanoparticle clouds may be formed all along the defects instead of chains? ? Welldefined systems with a number of oriented topological de- 
fects are thus highly desirable, also for a future control of anisotropic nanoparticle assemblies.

Smectic liquid crystals can form ordered patterns of defects, in $1 \mathrm{D}$ or $2 \mathrm{D}$, depending upon either the symmetry of the underlying substrate? ?, or the film thickness? ? ? and applied

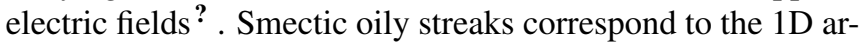
rays, formed in thin films at the interface between a substrate imposing unidirectional planar anchoring (i.e. parallel to the substrate) of the liquid crystal molecules and a medium such as air that imposes homeotropic anchoring (i.e. perpendicular to the interface). It has been shown on crystalline substrates that such hybrid anchoring induces the bending of the smectic layers into a periodic array of flattened hemicylinders, which are parallel to the substrate and perpendicular to the planar anchoring direction? ? ?. On $\mathrm{MoS}_{2}$, the region surrounding the axis of curvature of the bent layers, where the distortion energy is high, appears replaced by a rotating grain boundary $(\mathrm{RGB})^{\text {? }}$. First, is the RGB a generic feature for smectic liquid crystals with similar elastic constants, independent of the substrate? Since RGBs are expected to contain parallel edge dislocations? ${ }^{?}$, this would allow linear nanoparticle assemblies to form on a wide class of substrates. Second, how are the flattened hemicylinders connected to the substrates: does the connective region require new kinds of defects? To solve precisely the structure on rubbed polyvinyl alcohol polymer (PVA), we have combined optical microscopy, X-ray diffraction and ellipsometry on smectic liquid crystal films of 4-noctyl-4'-cyanobiphenyl (8CB). We have localized a number of dislocations, parallel to the hemicylinders, in relation with the shape of the grain boundaries in which they are involved, coexisting in the oily streaks with melted 2D defects. These results allowed the interpretation of the evolution of the period of the flattened hemicylinders arrays as a function of thickness (Figure ??), revealing in the same time a particularly large zenithal smectic anchoring energy on rubbed PVA.

\section{Materials and methods : Optical Microscopy and $\mathrm{X}$-ray Experiments}

We have prepared an $8 \mathrm{CB}$ film on rubbed PVA substrate by spin-coating of a toluene solution (concentration $0.1 \mathrm{M}$, acceleration $400 \mathrm{rpm} / \mathrm{s}$ and speed $4000 \mathrm{rpm}$ ). The samples were first observed by optical microscopy. Figure ?? presents the typical texture of a thin $8 \mathrm{CB}$ film observed in a reflection mode between crossed polarizers. The alternating bright and dark stripes correspond to domains containing molecules almost parallel or perpendicular to the substrate respectively (i.e. perpendicular or parallel smectic layers respectively). This texture has been evidenced on crystalline substrates, $\mathrm{MoS}_{2}$ and mica. It has been interpreted by a stacking of the smectic layers in periodic hemicylinders (schematic on Figure ??), the

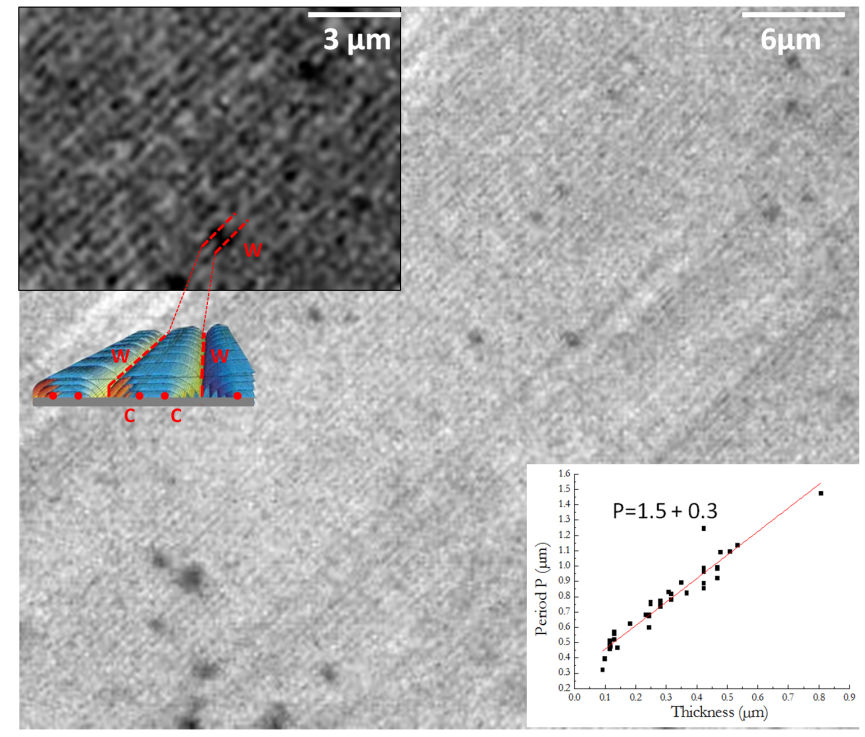

Fig. 1: 8CB/PVA Optical Microscopy image in reflection mode between crossed polarizers. Small stripes (hemicylinders in top view) with period $500 \mathrm{~nm}$ are visualized for a film thickness of $120 \mathrm{~nm}$. Schematic view of the flattened hemicylinders is also shown. Inset graph: stripes period as a function of the film thickness. The red line is a linear fit.

period of the hemicylinders being determined through the period of the stripes observed by an optical microscope? ? . It occurs on PVA as well, a substrate similarly inducing planar but also unidirectional anchoring. Polarized optical microscopy in reflection mode (between parallel polarizers) allows to determine the thickness of the smectic film through the measurement of the Newtons' tints. The evolution of the stripe period as a function of the thickness is presented on the insert of Figure ??. Above the thickness $120 \mathrm{~nm}$, it is linear with a slope 1.5 and an offset of $300 \mathrm{~nm}$. The period is consequently larger than twice the thickness, for all observed thicknesses. The surface morphology of the stripes corresponds to the one of flattened hemicylinders. This was already evidenced on $\mathrm{MoS}_{2}$ substrates ${ }^{?}$, but the slope (4.4) and offset $(500 \mu \mathrm{m})$ were different, demonstrating an influence of the anchoring characteristics (i.e. substrates properties) on the stripes period ${ }^{?}$.

$\mathrm{X}$-ray diffraction measurements were carried out at the SIXS beamline and the D2AM beamline on the SOLEIL synchrotron and on the ESRF synchrotron facilities respectively. On SIXS, the photon energy was fixed to $18 \mathrm{keV}$ and the Xray beam size to $300 \times 300 \mu \mathrm{m}^{2}$. In a grazing incidence set-up (with an incident angle of $0.2^{\circ}$ ), the stripes observed by optical microscopy are oriented parallel to the incident beam that probes a strip of the film of width $300 \mu \mathrm{m}$ and length equal to the sample size, $1 \mathrm{~cm}$. A scattered ring is observed on the $2 \mathrm{D}$ XPAD detector located at $1900 \mathrm{~mm}$ from the sample (figure 

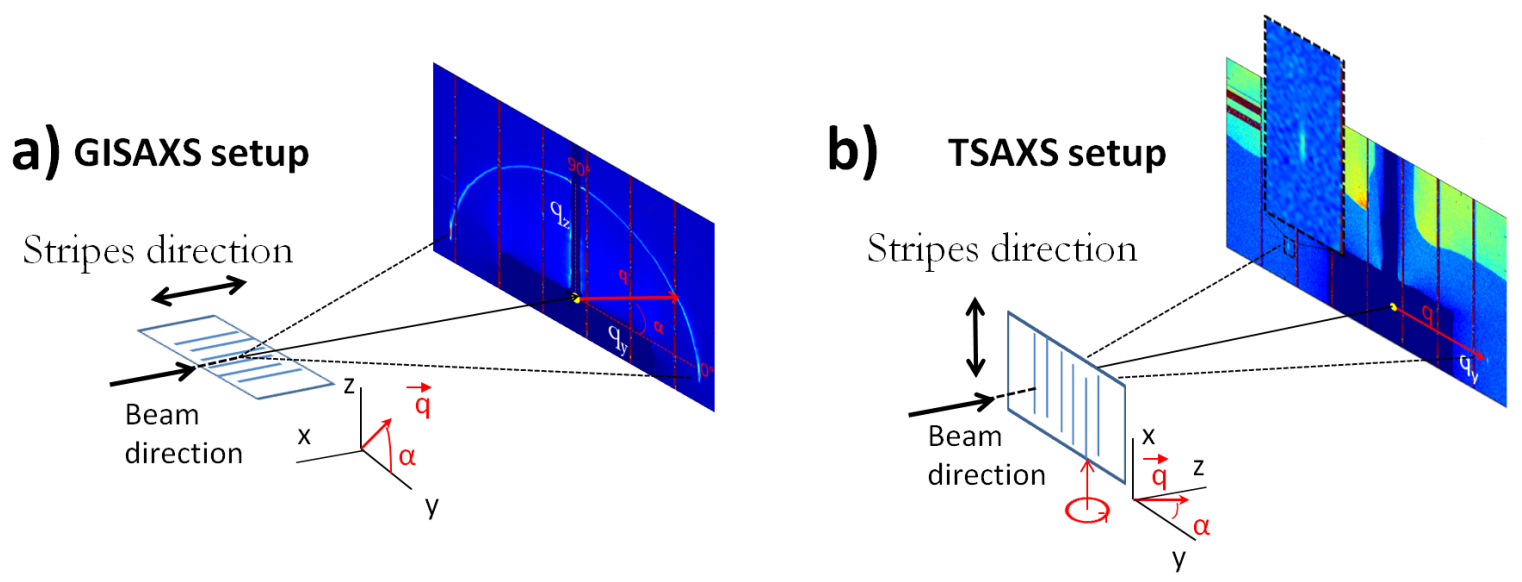

Fig. 2: a) Grazing incidence small angle X-ray scattering (GISAXS) setup and image of the scattered ring on the XPAD detector. b) Transmission small angle X-ray scattering (TSAXS). Two diffracted brackets-like features are visible on the XPAD detector.

??a). This ring is associated with the projection of the wave vector transfer, $\mathbf{q}$ in the $2 \mathrm{D}$ detector plane. The radius of the ring is $q=0.2 \AA^{-1}$. It corresponds to the expected absolute value of the $8 \mathrm{CB}$ wave vector transfer, smectic layers period being $\mathrm{d}=31.6 \AA^{\text {? }}$. This observation is in agreement with the wave vector transfer being parallel to the $2 \mathrm{D}$ detector plane. The scattered intensity is continuously defined from $\alpha=7^{\circ}$ to $\alpha=173^{\circ}, \alpha$ being the tilt of the wave vector transfer with respect to the substrate. For both extreme values, $\mathbf{q}$ is quasiparallel to the substrate and for $\alpha=90^{\circ}, \mathbf{q}$ is perpendicular to the substrate. $\alpha \leq 7^{\circ}$ and $\alpha \geq 173^{\circ}$ correspond to an exit angle of $0.17^{\circ}$ for the scattered beam. This is the value of the critical angle for the $8 \mathrm{CB} /$ air interface, preventing the possibility of observing photon below, respectively above, such a value ? ? Moreover the scattered signal at $90^{\circ}$ is superimposed to the intense substrate and film reflectivity. It can not be measured directly and is hidden by a beam stop. This excludes the measurements of $80^{\circ} \leq \alpha \leq 100^{\circ}$. To obtain the quasi-complete distribution of rotating $\mathbf{q}$ as a function of $\alpha$ and in particular to probe the interface with substrate, we have also used a set-up in transmission geometry. In this latter set-up, the sample is rotated by $90^{\circ}$ and is perpendicular to the incident X-ray beam (Figure ??b). The probed area is then reduced to $300 \mu \mathrm{m} \times$ $300 \mu \mathrm{m}^{2}$ and the scattered signal becomes associated with $\mathbf{q}$ parallel to the interface. In order to probe the rotation of $\mathbf{q}$, the sample can be rotated from $-10^{\circ}$ to $30^{\circ}$ about the alpha axis. The scattered signal appears as two brackets perpendicular to the stripes on the 2D detector (Figure ??b), of constant shape, confirming that the wave vector transfer rotates perpendicular to the stripes.

The transmitted and reflected X-ray signals were combined after a removing of the background to obtain the integrated along q intensity variation, from $-10^{\circ}$ to $80^{\circ}$, normalized to the
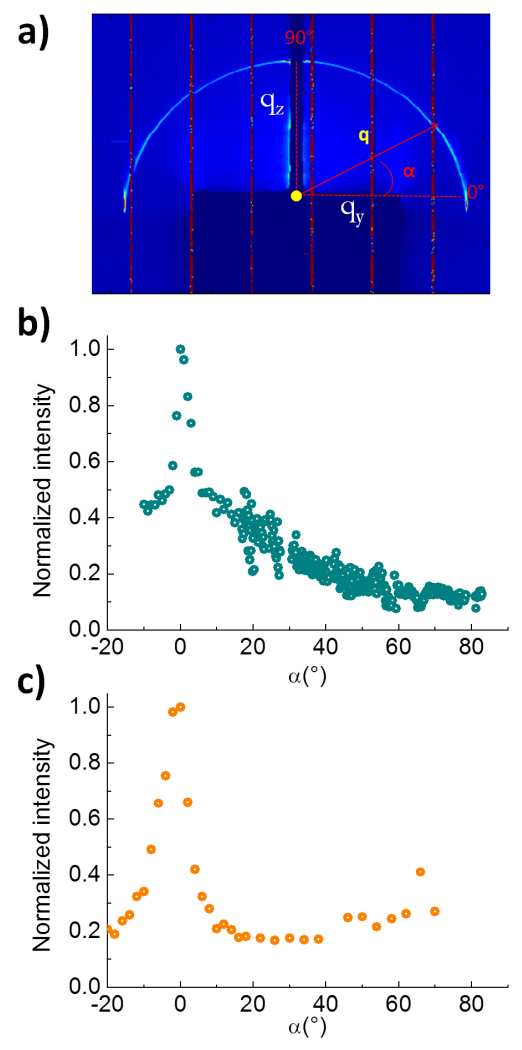

Fig. 3: a) Grazing incidence small angle X-ray scattering (GISAXS) image of the scattered ring on the XPAD detector, for an 8CB thickness of $100 \mathrm{~nm}$, on rubbed PVA substrate. b) Normalized integrated intensity for the 8CB film of thickness $100 \mathrm{~nm}$, obtained combining GISAXS signal of Figure ??a and TSAXS signals. c) Normalized integrated intensity for a $8 \mathrm{CB}$ film of $230 \mathrm{~nm}$ thickness obtained in TSAXS configuration. 
maximum intensity at $\alpha=0^{\circ}$, presented in Figure ??b. The absence of discontinuities in the data suggests that the average probed film thickness in the grazing incidence set-up is equal to the one in the transmission geometry.

On the ESRF D2AM beamline, the X-ray photon energy was fixed to $11.3 \mathrm{keV}$. We used a transmission set-up only, associated with a sample rotation from $\alpha=-20^{\circ}$ to $\alpha=70^{\circ}$. A correction taking into account the variation of the substrate (glass+PVA) X-ray absorption during the sample rotation has been applied. The corresponding X-ray scattered signal, after removing of the background and integration along $\mathrm{q}$ and along the bracket, is shown on Figure ??c normalized to the maximum intensity at $\alpha=0^{\circ}$.

Ellipsometric measurements of the Mueller matrix elements of an 8CB film on rubbed PVA substrate were performed on a CCD camera placed in the Fourier plane of the $\times 100$ microscope objective of a home-made micro-ellipsometry setup. This allows to perform measurements on small areas $(50 \mu \mathrm{m} \times$ $50 \mu \mathrm{m})$ of well-defined thickness. The detector provides a $2 \mathrm{D}$ image of the reflected intensity where the polar $\left(0^{\circ}<\theta<60^{\circ}\right)$ and azimuthal $\left(0^{\circ}<\phi<360^{\circ}\right)$ angles of the beam at the surface are mapped into the cartesian axes $x$ and $y$ of detector, with $x=\sin (\theta) \times \cos (\phi)$ and $y=\sin (\theta) \times \sin (\phi)$. The Mueller matrix elements are extracted from 16 intensity images recorded for different combinations of polarization states generated for the incident beam and filtered for the emerging beam. All Mueller matrix elements were normalized to the total reflected intensity given by the first matrix element $\mathrm{m}_{11}$.

\section{X-ray analysis}

\subsection{Thin film.}

A thin sample, of average thickness of $100 \mathrm{~nm}$ presenting thickness variations between 70 and $130 \mathrm{~nm}$ over typical length scales of $200 \mu \mathrm{m}$, was first studied (SOLEIL SIXS beamline). The X-ray scattering measurements can be first interpreted qualitatively to analyze the general organization of the $8 \mathrm{CB}$ smectic layers in the flattened hemicylinders.

We assume that, considering the weak amount of matter contributing to the scattered signal, the kinematic approximation can be applied. Therefore, for a given $\alpha$, the integrated intensity $\mathrm{I}(\alpha)$ is proportional to the number, $\mathrm{n}(\alpha)$, of smectic layers, whose normal, parallel to the wave-vector transfert, $\mathbf{q}$, makes an angle $\alpha$ with the substrate. Figure ??b then shows the distribution of smectic layers versus $\alpha$, deduced from $\mathrm{I}(\alpha)$ ( $\alpha=0^{\circ}$ corresponds to smectic layers perpendicular to the substrate and the value $\alpha=80^{\circ}$ corresponds to smectic layers quasi-parallel to the substrate). The observation that $\mathbf{q}$ belongs to the plane perpendicular to the stripes and that quasi-all possible orientations of $\mathbf{q}$ can be observed (Figure ??a), with $\alpha$ ranging from $0^{\circ}$ to $80^{\circ}$, is fully consistent with a continu- ous curving of the smectic layers within stacked hemicylinders with little elastic deformation of the smectic layers. However, an array of perfect flattened hemicylinders, as schematically depicted on Figure ?? would yield $\mathrm{I}(\alpha)$ constant for $\alpha \leq 80^{\circ}$. In contrast, Figure ??b shows a smooth decrease of $\mathrm{I}(\alpha)$ when $\alpha$ increases up to $80^{\circ}$. The scattered signal also shows a peak around $\alpha=0^{\circ}$, two times larger than the surrounding signal. This latter observation can be explained by the fact that, when one observes the scheme of Figure ??, the scattered signal of smectic layers perpendicular to the interface is doubled with respect to those that contribute to the intensity for larger $\alpha$ values: indeed, when $\alpha=0^{\circ}$, both layers that bent towards right or towards left are contributing to the signal, while for alpha non zero they give two separate contributions. In addition, the observation of decreasing $\mathrm{I}(\alpha)$ above $\alpha=10^{\circ}$ evidences a number of rotating smectic layers decreasing when $\alpha$ increases. It can be interpreted as if curved smectic layers were removed from perfect hemicylinders. A higher number of rotating layers at large $\alpha$ is removed with respect to small $\alpha$. Since the curved layers close to the axis of curvature ( $\mathrm{C}$ on Figure ??) are the most distorted ones, we expect them to be removed and replaced by an extension of the parallel smectic layers from the center of the flattened hemicylinder. This corresponds to the presence of a rotating grain boundary (RGB), joining parallel smectic layers to curved ones, which has already been reported in $8 \mathrm{CB}$ films on $\mathrm{MoS}_{2}$ substrates?

The determination of $\mathrm{I}(\alpha)$ for a large range of $\alpha$ values, thanks to the combination of reflection and transmission setups allows to quantitatively determine the profile for the RGB in relation to the presence of dislocations. We have used the geometrical model previously developped to describe the array of hemicylinders (Figure ??a) observed on $\mathrm{MoS}_{2}$ substrates ${ }^{?}$. The hemicylinders have been shown as joined by curvature walls (W on Figure ??a), defined by $\omega$, the angle related to their height (see Figure ??a). According to this model, the distribution of smectic layers, $n(\alpha)$, related to their orientation $\alpha$, is :

$$
n(\alpha)=\left\{\begin{array}{cc}
\frac{1}{d}\left(e \frac{\cos (\omega)}{\cos (\alpha)}-r(\alpha)\right) & \text { when } \alpha \leq \omega \\
\frac{1}{d}(e-r(\alpha)) & \text { when } \alpha \geq \omega
\end{array}\right\}
$$

where $e$ is the overall thickness, $r(\alpha)$ is the 2D profile of the RGB expressed in polar coordinates, the origin being the axis of curvature of the hemicylinders; $d$ is the smectic period (Figure ??a). In other terms, $r(\alpha) / d$ represents the number of smectic layers that are 'lacking' from the stack of layers whose normal is oriented with the angle $\alpha$. The scattered intensity being proportional to the distribution of rotating smectic layers, we can write:

$$
I(\alpha)=\left\{\begin{array}{cc}
A\left(e \frac{\cos (\omega)}{\cos (\alpha)}-r(\alpha)\right) & \text { when } \alpha \leq \omega \\
A(e-r(\alpha)) & \text { when } \alpha \geq \omega
\end{array}\right\}
$$




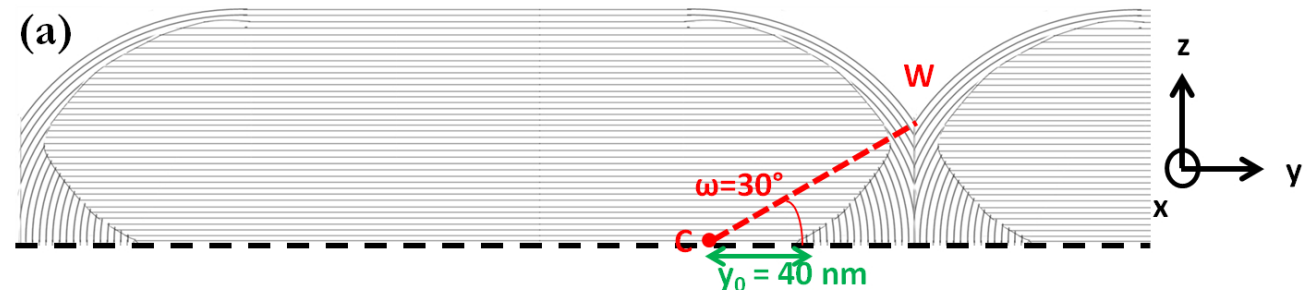

(b)

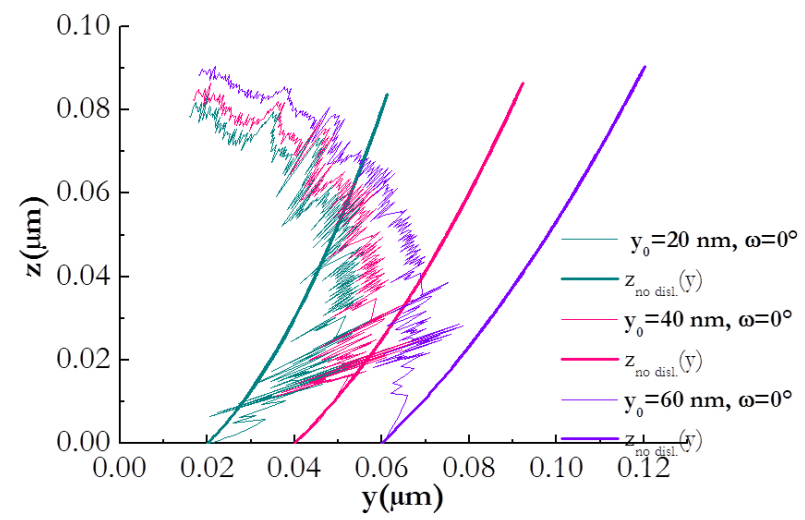

(d)

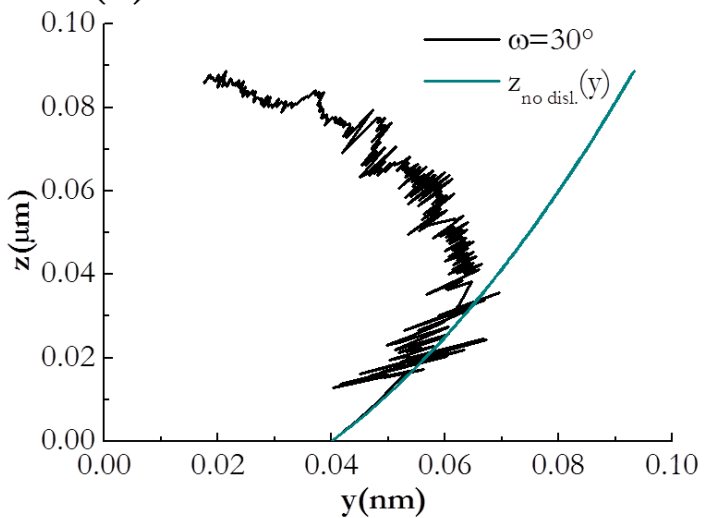

(c)

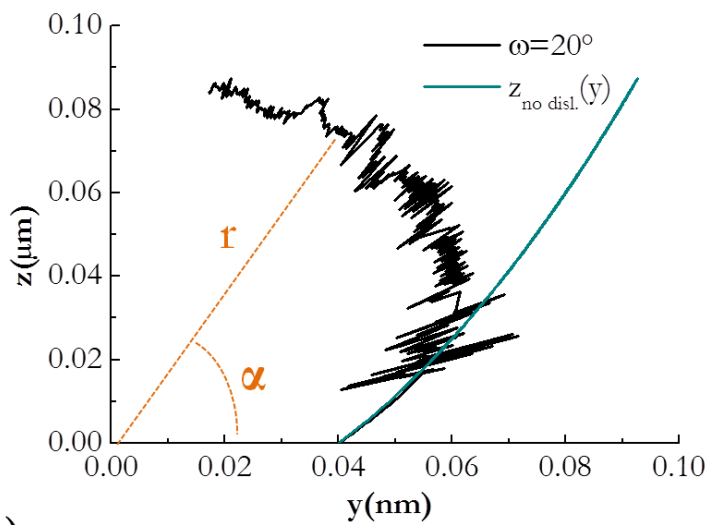

(e)

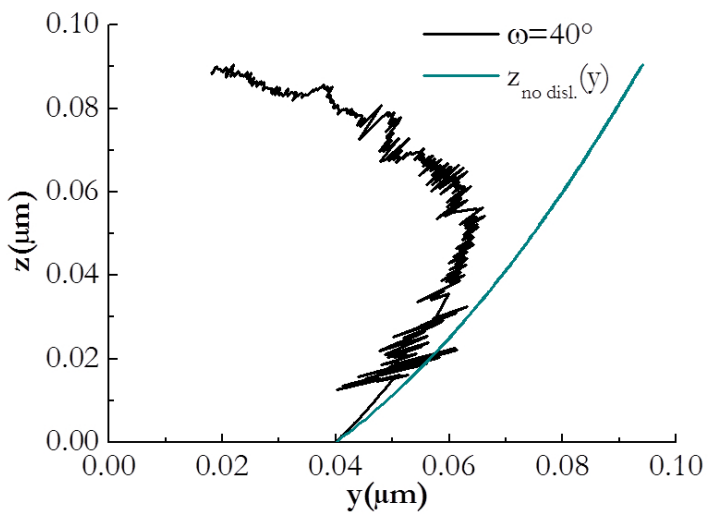

Fig. 4: a) Schematic of the rotating grain boundaries of oily streaks in side view. The smectic layers are represented and become periodic lines due to the side view, of period $d=3.16 \mathrm{~nm}$. The angle $\omega$ is between the substrate plane, the curvature axis, $\mathrm{C}$ and the top of the curvature wall, $\mathrm{W}, \omega=30^{\circ}$. b) -e) Plot of the RGB profiles, $\mathrm{r}(\alpha)$ (eq. ??). The profiles are calculated for different values of $\mathrm{y}_{o}\left(\mathrm{y}_{o}\right.$ distance is shown in (a)) and $\omega$. b) $y_{o}=20,40,60 \mathrm{~nm}$ for $\omega=0^{\circ}$; c), d), e) $y_{o}=40 \mathrm{~nm}$ and $\omega=20,30$ and $40^{\circ}$ respectively. The curve $\mathrm{z}_{n o-d i s l}(\mathrm{y})$ in each graph corresponds to a theoretical profile of grain boundary without dislocations (eq. ??), for each $y_{o}$ value. 
Then the profile of the RGB is obtained by:

$$
r(\alpha)=\left\{\begin{array}{cc}
\left(e \frac{\cos (\omega)}{\cos (\alpha)}-\frac{I(\alpha)}{A}\right) & \text { when } \alpha \leq \omega \\
\left(e-\frac{I(\alpha)}{A}\right) & \text { when } \alpha \geq \omega
\end{array}\right\}
$$

Two parameters remain unknown, $\omega$ and $A$. To solve this problem, we assume the lowest possible energetic cost for the RGB. It may be composed of the smallest possible number of dislocations, for the connection between the curved smectic layers and the horizontal smectic layers. One can calculate the form of a grain boundary without dislocation. The following equation is obtained? :

$$
z_{n o-d i s l}(y)=\frac{y^{2}-y_{o}^{2}}{2 y_{o}}
$$

where $y_{o}=r\left(\alpha=0^{\circ}\right)=\left(e \cos (\omega)-I\left(\alpha=0^{\circ}\right) / A\right)$ represents the position of the bottom of the RGB. For a given value of $\omega$ and $y_{o}, A=I\left(\alpha=0^{\circ}\right) /\left(e \cos (\omega)-y_{o}\right)$. Figures ??b-d represent the RGB profiles calculated either from (3) or from (4). The curves are calculated for different $\omega$ and $y_{o}$ values. One can observe in figure ??b that, for $\omega=0^{\circ}$, the value $y_{o}=40$ $\mathrm{nm}$ gives a good agreement between the two kinds of grain boundaries, in the region close to the substrate (low $\mathrm{z}$ values). This is also true if we perform the comparison for other $\omega$ values. One can then fix $y_{o}$ to $40 \mathrm{~nm}$ and search for the $\omega$ value that presents the best agreement (Figure ??c-d-e). One can observe that for $\omega$ higher than $30^{\circ}$ the curves do not match, meaning that omega is lower than $30^{\circ}$. A close inspection also reveals that it better matches for $\omega=20^{\circ}$ and $30^{\circ}$ than for $\omega=0^{\circ}$. We thus not only obtained the profile of the RGB buried close to the axes of curvature of the hemicylinders but also the $\omega$ value around $20^{\circ}-30^{\circ}$ associated with the curvature walls of these $8 \mathrm{CB}$ films of thickness $100 \mathrm{~nm}$. The model of curved smectic layers shown on Figure ??a is finally obtained for thin films, of thickness around $100 \mathrm{~nm}$, associated with a large area in the center of the flattened hemicylinders of unfavorable anchoring (smectic layer parallel to the substrate).

\subsection{Thick film}

A film of thickness between 210 and $230 \mathrm{~nm}$, has been measured (ESRF D2AM beamline). The signal evolves with respect to $100 \mathrm{~nm}$ thin films, as shown by Figure ??c. We observe a larger increase of the intensity at $\alpha=0^{\circ}$, as compared with the one of the curved layers $\left(10^{\circ} \leq \alpha \leq 70^{\circ}\right)$. The intensity is then almost constant between $\alpha=10^{\circ}$ and $\alpha=45^{\circ}$, and slightly increases at about $50^{\circ}$, to be quasi-constant again between $\alpha=50^{\circ}$ and $70^{\circ}$. Apart from the signal at $\alpha=0^{\circ}$, the intensity evolution becomes closer to the one associated with perfect hemicylinders joined by curvature walls. This observation can be interpreted by a proportion of the signal attributed to the RGB that diminishes when the overall thickness increases. This suggests that the RGB may not vary when the film thickness increases. In agreement with previous results obtained on $\mathrm{MoS}_{2}$ substrates, it can be explained by a RGB resulting from the balance between elastic energy of smectic layers with very high curvature radius (close to the curvature axis) and the energy cost of the dislocations still present at the top of the rotating grain boundary. Both are independent of the film thickness. The measured X-ray intensity increasing around $\alpha=50^{\circ}$, followed by a constant signal for higher $\alpha$, may hint toward an $\omega$ value, characteristic of the curvature wall height around $50^{\circ}$.

The ratio $\rho$ of the integrated additional peak intensity at $\alpha=0^{\circ}$ (Figure ??c) over the overall integrated intensity (subtracted from the additional peak at $\alpha=0^{\circ}$ ) amounts to $\rho=$ 0.3 , evidencing a strong increase of the relative number of smectic layers perpendicular to the substrate. The additional perpendicular layers are most likely located close to the PVA substrate, which is known to favor unidirectional planar anchoring. This $\rho$ value may indicate the presence of a transition sublayer connecting to the substrate the upper sublayer, which includes the curved smectic layers at the hemicylinder border and the parallel smectic layers in the hemicylinder center. The presence of this transition sublayer may prevent from the violation of the planar anchoring on the substrate by the parallel smectic layers. Two assumptions can be proposed for the structure of this transition sublayer. First, it can be constituted of a grain boundary joining parallel and perpendicular smectic layers ( see Figure ??a-b, the grain boundary being indicated in green). Second, it can be composed of an homogeneous sublayer of smectic perpendicular layers (Figure ??c).

For the first assumption, two extreme models can be built to account for the presence of these perpendicular layers. They are schematized on Figure ??a-b. The transition sublayer is divided between vertical and flat smectic layers, joined by a grain boundary (green in Figure ??a-b). Its structure may be determined by two factors: the amount of dislocations needed to connect smectic layers across the grain boundary and the anchoring energy of $8 \mathrm{CB}$ on rubbed PVA that favors vertical smectic layers. The first factor tends to create a grain boundary of tilt $45^{\circ}$ with respect to the substrate (Figure ??a). This would avoid the presence of dislocations to the detriment of a large area of parallel 8CB layer on rubbed PVA in the center of the flattened hemicylinders (Figure ??a). The second factor tends to tilt the grain boundary by less than $45^{\circ}$, in order to cover the rubbed PVA as much as possible with vertical smectic layers. The latter situation would introduce a number of distributed dislocations, depending on the tilt of this grain boundary, and on the thickness of this transition sublayer (Figure??b). For the two extreme models shown in Figures ??a and ??b, with $\rho=0.3$, thicknesses of $49 \mathrm{~nm}$ and 30 $\mathrm{nm}$ are respectively obtained for the sublayer.

The second assumption would be associated with the presence 
of a central 2D defect between the perpendicular smectic layers of the transition sublayer and the central horizontal smectic layers (see Figure ??c). No connection between these two kinds of smectic layers being possible, the 2D defect may be melted into a nematic area of thickness not too small to account for a $90^{\circ}$ rotation of the corresponding nematic director. If we consider the thickness of this nematic area of the order of $10 \mathrm{~nm}$, the ratio $\rho=0.3$ leads to a thickness equal to $17 \mathrm{~nm}$ for the transition sublayer made of homogeneous perpendicular layers.

\section{Model of the internal film structure}

\subsection{Ellipsometry.}

In order to confirm the occurrence of a transition sublayer with smectic layers perpendicular to the substrate for films of thickness larger than $100 \mathrm{~nm}$, we present the results of the ellipsometry measurements on a film with an intermediary thickness of $170 \mathrm{~nm}$. The actual thickness of $170 \mathrm{~nm}$ of the film was determined using the value of the period of the oily streaks measured in the direct plane of the microscope objective where the surface was imaged. ${ }^{?}$. Ellipsometry is well complementary to X-ray diffraction for the analysis of birefringent thin films thanks to its sensitivity to the variation of birefringence along the thickness of the film.

Figure ??(d) presents the Mueller matrix elements measured on the $170 \mathrm{~nm}$ thick film? ${ }^{\text {? }}$. The symmetries of the Mueller matrix elements are representative of the mirror symmetry of the oily streaks with respect to the center of the hemicylinders. The elements of the two off-diagonal blocks $\left(\mathrm{m}_{i j}\right.$ with $(i j)=(13)$, (14), (23) and (24)) are related to transfers of polarization which are mainly associated with the in-plane optical anisotropy of the thin films.

To analyze quantitatively the data, it is necessary to compare them to calculated ones. We have recently developed a model based on the organization of the smectic layers in oily streaks to calculate the Mueller matrix $\mathbf{M}$ of thin films containing oily streaks. We have considered the two extreme schematized models shown on Figures ??a-b (assumption 1) ${ }^{\text {, }}$, or the model of homogeneous perpendicular smectic layers (assumption 2, Figure ??c). In these models, the oily streaks are approximated by sublayers containing domains with constant orientation of smectic layers, hence constant optic axes orientations, as shown on Figures ??a-c). The equivalent anisotropic permittivity of each sublayer was obtained from the average of the optical constants of each domain, weighted by their respective volume fractions, along each direction of the cartesian referential. This yields a multilayer model with graded anisotropy. The difference between the two first models (Figures ??a-b) lies in the fraction of perpendicular smectic layers covering the substrate in the transition sublayer.
It varies between the minimum fraction, corresponding to a grain boundary tilted at $45^{\circ}$ (Figure ??a) and the maximum fraction, corresponding to a complete covering of the substrate (Figure ??b). A fit of the experimental Mueller matrix elements has been performed with the fraction of perpendicular smectic layers in the transition sublayer used as varying parameter. A minimum thickness of the transition sublayer was necessary to obtain a good agreement between experimental and calculated data. For transition sublayer thickness of 40 $\mathrm{nm}$, a complete coverage of the substrate by perpendicular layers is obtained from the fit to the data and a reasonable agreement between experiments and simulations is obtained?

In order to compare assumption 1 and 2, we have considered the third model of Figure ??c. We have fitted the experimental data with this model by varying the thickness of the transition sublayer, the overall thickness remaining constant. The best fit is obtained for a thickness $25 \mathrm{~nm}$, including the transition sublayer together with the nematic 2D defect. In this case, the agreement with experimental data is really good, strongly suggesting that the transition sublayer is composed by homogeneous perpendicular smectic layers separated from the horizontal ones by a nematic 2D defect. Moreover the good agreement further confirms a structure for the upper sublayer made of curved smectic layers, in presence of a RGB ${ }^{\text {? }}$.

For the $8 \mathrm{CB}$ films, of thickness $100 \mathrm{~nm}$, the number of perpendicular layers has been found negligible. We thus expect in these films the presence of a large area with an unfavorable parallel smectic layer on the substrate, at the center of the hemicylinders (Figure ??a). Ellipsometry measurements show that this unfavorable parallel smectic layer disappears in films thicker than $170 \mathrm{~nm}$, thanks to the establishment of a transition sublayer close to the substrate, associated with a melted grain boundary between perpendicular and parallel smectic layers. We can expect that, above $170 \mathrm{~nm}$, the anchoring still favors a complete covering of the rubbed PVA by perpendicular layers. This is in agreement with the intense peak observed by X-rays at $\alpha=0^{\circ}$, for a thickness $210-230 \mathrm{~nm}$, also corresponding to a transition sublayer thickness of the order of $20 \mathrm{~nm}$.

\subsection{Evolution of the internal structure of the oily streaks.}

Combining ellipsometry and X-ray measurements, we evidence that a transition sublayer with thickness of the order of $20 \mathrm{~nm}$ may be sufficient to avoid anchoring breakage due to parallel smectic layers reaching the substrate. In addition, the sublayer may not evolve when the overall thickness increases, the same value being found for thicknesses 170 and $220 \mathrm{~nm}$. We are now able to propose a model for the internal structure of the oily streaks, including a description of the upper and lower sublayers as depicted on figure ??a for the film thickness $e$ between 210 and $230 \mathrm{~nm}$. Figure ??b presents the $\mathrm{X}$-ray scattering signal calculated for the corresponding film 
(a)

(b)

(c)

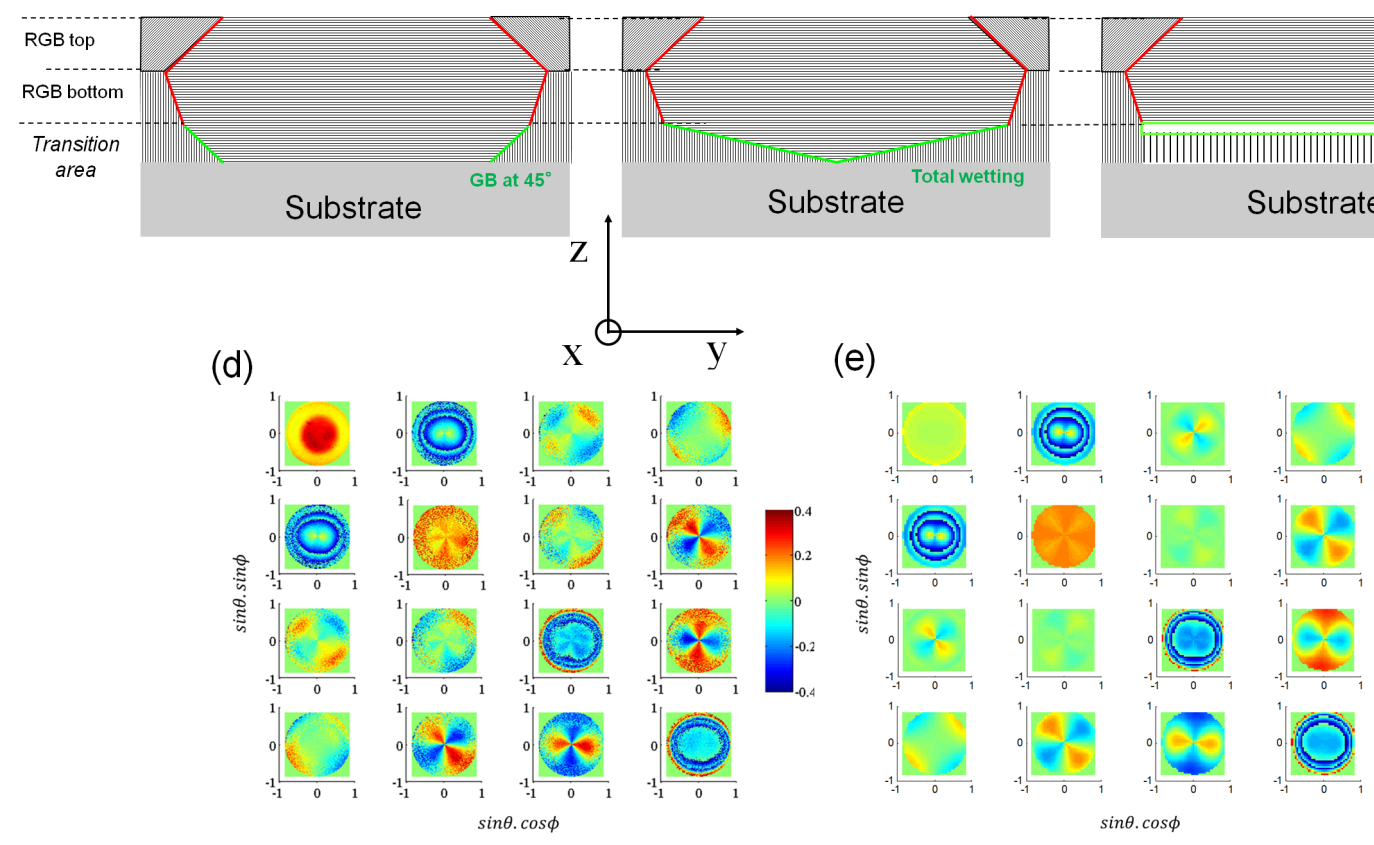

Fig. 5: Above : Schematic model (the curving of the smectic layers is schematically represented by the two different orientations of the smectic layers, the RGB being represented in red), for a film thickness $170 \mathrm{~nm}$, (a) associated with a transition sublayer in presence of a grain boundary, shown in green, with no dislocation, but coexisting with an area of unfavorable anchoring; (b) associated with a transition sublayer with favorable anchoring but in presence of a grain boundary, shown in green, with edge dislocations distributed along the grain boundary; (c) associated with a transition sublayer with favorable anchoring but with a nematic 2D defect (in green). Below : (d) Measured Mueller matrix elements for a film with total thickness of $170 \mathrm{~nm}$, as mapped by the objective $(x=\sin \theta \cdot \cos \phi, y=\sin \theta$. $\sin \phi)$; (e) calculated Mueller matrix elements, for a film with total thickness of $170 \mathrm{~nm}$ and corresponding to the model shown on (c), with a transition sublayer thickness of $20 \mathrm{~nm}$, also including the nematic 2D defect . 
(blue curve). We assume, first, that the RGB profile is the same as that determined for a film of $100 \mathrm{~nm}$ (Figure ??c), second, that $\omega=50^{\circ}$ and, third, that the transition sublayer is of thickness $20 \mathrm{~nm}$, leading to a thickness between 180 and $200 \mathrm{~nm}$ for the upper sublayer, with curved smectic layers (taking into account the presence of a melted grain boundary of thickness $10 \mathrm{~nm})$. The calculated $I(\alpha)$ associated with curved smectic layers $\left(\alpha>10^{\circ}\right)$, of thickness $190 \mathrm{~nm}$, is presented on Figure ??b. It appears in very good agreement with the similarly normalized experimental data (Figures ??c and ??b). This confirms that when the thickness increases, starting from $e=100 \mathrm{~nm}$, the RGB does not vary and the $\omega$ value increases from $30^{\circ}$ to $50^{\circ}$. A transition sublayer of $20 \mathrm{~nm}$ is already formed for $e=170 \mathrm{~nm}$ and does not appear to significantly vary for $e=220 \mathrm{~nm}$. For $e=220 \mathrm{~nm}$, the ratio, $\rho$, suggests a thickness for the transition sublayer of $20 \mathrm{~nm}$, which leads to a thickness of $190 \mathrm{~nm}$ for the upper sublayer thickness, also fully consistent with the experimental X-ray scattered signal of curved smectic layers. (Figures ??c and ??b).

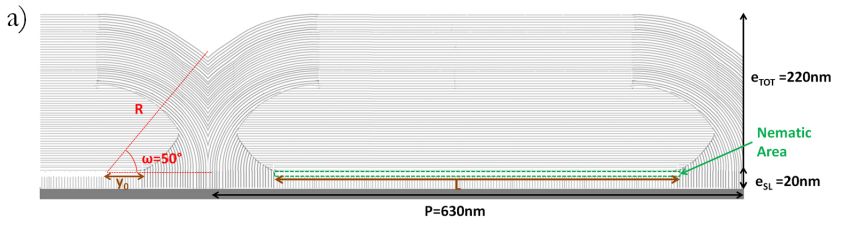

b)

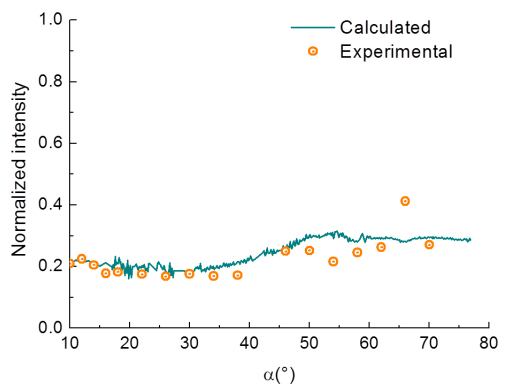

Fig. 6: $8 \mathrm{CB}$ film of thickness $220 \mathrm{~nm}$ : a) Full model of the internal $8 \mathrm{CB}$ structure. (b) Normalized intensity of the X-ray experimental signal (orange circles), compared to the calculated signal (blue line).

\section{Discussion}

The formation of linear oily streaks in smectic liquid crystal thin films deposited on rubbed PVA substrates is due to the competition between different mechanisms: anchoring at the 8CB-PVA and 8CB-air interfaces, minimization of the surface energy at the deformable 8CB-air interface and minimization of the defect energy, in particular the grain boundaries energy, which may be dominated by the presence of dislocation lines. The high $8 \mathrm{CB}$-air surface energy, $30 \mathrm{~mJ} / \mathrm{m}^{2}$, tends to flatten the 8CB-air interface and the overall shape of the linear domains, constraining the smectic layers to become organized in periodic flattened hemicylinders ? ? This stacking of smectic layers into hemicylinder connected by curvature walls between neighboring hemicylinders, has been demonstrated on crystalline substrate by a combination of optical microscopy, atomic force microscopy and X-ray diffraction? In the present work, we show that the competition between the different mechanisms results in the creation of an array of various topological defects. Grain boundaries are created, containing arrays of dislocations, all parallel to the hemicylinder axis (i.e. perpendicular to the anchoring direction on the PVA substrate), of different expected Burger vectors, together with a nematic 2D defect. It has recently been demonstrated that dislocation arrays can be used to organize and orient nanoparticles? ? We now evidence the localization of the dislocations and show the interplay between the structure of grain boundaries and the presence of dislocations.

First, dislocations parallel to the hemicylinder axis, are expected at the basis of the curvature walls between neighboring hemicylinders, where the disorientations between the smectic layers is the highest ${ }^{\text {? }}$. These dislocations are expected of various Burger vectors, depending on their localization along the curvature wall $^{\text {? }}$. Second, given the hemicylindrical stacking of the smectic layers, the presence of a RGB is the only possible structure close to the hemicylinder axis, consistent with Xray data and according to the hypothesis that the smectic layers of the highest curvature radius are eliminated, as already suggested on $\mathrm{MoS}_{2}$ substrates? ${ }^{\text {? }}$. This hypothesis is now validated on rubbed PVA substrates, by the additional agreement with ellipsometry measurements. The RGB size was expected independent on the thickness for crystalline $\mathrm{MoS}_{2}$ substrates? which is now confirmed also for PVA substrate (see section ??). The present work completes these past observations by experimentally determining the RGB shape and demonstrating that it is associated with the presence of dislocations, distributed along the RGB. The RGB shape shape however eliminates the dislocations from its basis, leading to dislocations, still parallel to the hemicylinder axis, but only concentrated at its summit (Figure ??a). Moreover, as shown by the models of Figure ??a and ??a, the dislocations are of large Burger vector, between 6 and 7. It is thus possible that they become joined into a giant dislocation, which can be more stable than a number of dislocations of smaller Burger vector ${ }^{\text {? }}$. We can not select between these two hypotheses, neither X-ray data, nor ellipsometry, being sensitive to the intimate structure of the dislocations.

For film thickness up to $100 \mathrm{~nm}$, a sizeable amount of $8 \mathrm{CB}$ molecules in the center of each flattened hemicylinder is anchored in the energetically unfavorable orientation perpendicular to the substrate, i.e. the smectic layers are horizontal to the polymer substrate. However, when the smectic film thick- 
ness is increased, X-ray and ellipsometry measurements consistently show that the planar anchoring condition is fulfilled over the entire PVA substrate (Figure ??a). A transition sublayer appears with thickness of the order of $20 \mathrm{~nm}$ and the very good agreement between $\mathrm{x}$-ray, ellipsometric data and the model of Figure ??a strongly suggests that the transition sublayer is made of homogeneous perpendicular layers, leading to the presence of a nematic 2D defect (in green on Figures ??c and ??a). It is therefore more favorable to create this defect than violating the planar anchoring. X-ray and ellipsometry data show that the transition sublayer thickness remains close to $20 \mathrm{~nm}$ when the film thickness varies between $170 \mathrm{~nm}$ and $230 \mathrm{~nm}$.

We are now able to interpret the variation of the period, $P$, as a function of the thickness, $e$ (Figure ??c). The variation becomes linear, $P=1.5 e+300$, for $e \geq 120 \mathrm{~nm}$, suggesting that the transition sublayer is fully established when the film thickness exceeds $120 \mathrm{~nm}$. Referring to Figure ??a, the period is such that $P=L+\left(2 R \cos \omega-2 y_{\circ}\right)=2 e \cos \omega+\left(L-2 y_{\circ}-\right.$ $\left.2 e_{s l} \cos \omega\right)$, where $e_{s l}$ is the transition sublayer thickness, $\mathrm{L}$ is the lateral size of the nematic $2 \mathrm{D}$ defect (the distance between consecutive points where the bottom of the RGB intersects the sublayer), $R=e-e_{s l}$ is the maximum radius of the curved layers, and $y_{\circ}=40 \mathrm{~nm}$ is the lateral distance between the RGB bottom and the axis of curvature (see Figure ??a and ??a). If we consider that $\omega$ is almost constant with the film thickness, varying between $40^{\circ}$ and $50^{\circ}$ when the thickness increases from $120 \mathrm{~nm}$ to $220 \mathrm{~nm}$, then $2 \cos \omega$ varies between 1.5 and 1.3 , close to the 1.5 slope of the $\mathrm{P}$ vs. thickness curve (Figure ??c). Therefore, $L-2 y_{\circ}-2 e_{s l} \cos \omega$ is also almost constant as the film thickness increases, $e_{s l}$ is constant and L consequently only slightly increases from $430 \mathrm{~nm}$ to $460 \mathrm{~nm}$. When the overall thickness, $e$, increases, the lateral size of the nematic 2D defect within one hemicylinder does not vary, the period, $P$, increases mainly due to the addition of new curved smectic layers, yielding an increase of $R$.

The observed full coverage of the substrate by perpendicular layers means that the anchoring energy on the substrate is large enough to impose the establishment of a nematic 2D defect, providing that the film thickness is larger than $120 \mathrm{~nm}$. A nematic 2D defect of width $L$ is preferred with respect to a parallel smectic layer directly adsorbed on the substrate, along a length $L$. In order to estimate the corresponding low limit value for the anchoring energy on PVA substrate, the nematic 2D defect energy per unit of surface can be estimated. Two terms are expected : First the Landau-de Gennes penalty associated with the energy density of a melted volume in the nematic phase times the thickness, $\delta$ of the melted area: $E_{N G B}=\delta \times 3.10^{6}{\mathrm{~J} . \mathrm{m}^{-3}}$, for $8 \mathrm{CB}$ at $25^{\circ} \mathrm{C}^{?}$; Second the nematic distortion, associated with the $\pi / 2$ tilt of the nematic director, equal to $(K / 2)(\pi / 2)^{2} / \delta^{?}$, with $K=7.10^{-11}$ $\mathrm{J} . \mathrm{m}^{-1}$, the curvature elastic constant of $8 \mathrm{CB}^{?}$. At $25^{\circ} \mathrm{C}$, the thickness, $\delta$, that minimizes these two energies is $\delta=5.3 \mathrm{~nm}$, a very small value, for which the calculation of nematic distorsion may be not really accurate. It appears reasonable to consider it to range between 5 and $10 \mathrm{~nm}$, leading to a total energy per unit of surface between $3.210^{-2} \mathrm{~J}^{-\mathrm{m}^{-2}}$ and $3.910^{-2} \mathrm{~J}_{\mathrm{m}} \mathrm{m}^{-2}$. Since formation of the nematic $2 \mathrm{D}$ defect is more favorable than a violation of the planar anchoring, its energy per unit of surface corresponds to a low limit of the $8 \mathrm{CB}$ zenithal anchoring energy on rubbed PVA. The obtained low limit of the zenithal anchoring energy, of the order of $310^{-2}$ $\mathrm{J} . \mathrm{m}^{-2}$, appears much higher than the values reported for the zenithal anchoring energies of most nematics, usually smaller than $10^{-3} \mathrm{~J} . \mathrm{m}^{-2}$ ? ? . To the best of our knowledge, an anchoring energy of similar order of magnitude has been reported for nematics, only when nanometric films are concerned and, consequently, the first molecular layers are measured ? ${ }^{\text {? The }}$ zenithal anchoring energy associated with the first molecular layers may be the relevant energy for zenithal smectic energies, in contrast with nematic films. Indeed, it appears of the same order as the low limit estimated also for $8 \mathrm{CB}$ in unidirectional planar anchoring, but on crystalline substrates, $\mathrm{MoS}_{2}$ and mica? ? This suggests that, generally speaking, smectic zenithal anchoring energies are high, at least when they are associated with planar unidirectional anchoring.

It is interesting to note that the nematic 2D defect may be formed not only to allow for a complete covering of the substrate by perpendicular layers, but also, again, to avoid dislocations. The other possible assumption (first assumption) indeed corresponds to two facing grain boundaries (in green on Figures ??b) which would be entirely made of edge dislocations, for a complete covering of the substrate by perpendicular smectic layers. For exemple, for $L=460 \mathrm{~nm}$ and $e_{s l}=30$ $\mathrm{nm}$ (it corresponds to the $20 \mathrm{~nm}$ of assumption 2, together with $10 \mathrm{~nm}$ of greennematic 2D defect), there are, for each facing grain boundary, 9 dislocations of Burger vector 7, leading to an energy of $2 \times 9 \times 7 \times e_{d i s l}$. We consider that the energy of a dislocation is proportional to the Burger vector? ?

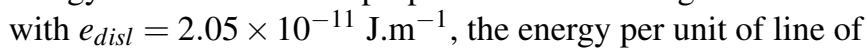
an elementary edge dislocation, previously measured in freestanding films of $8 \mathrm{CB}^{\text {? }}$. To calculate the energy of these two facing grain boundaries, one should also take into account an energy of grain boundary, proportional to the length of the grain boundaries, associated with the disorientation by $90^{\circ}$ of the smectic layers from each side of the grain boundary: $2 e_{G B} \sqrt{1 / 4(L)^{2}+e_{s l}^{2}}$, with $e_{G B}$ the grain boundary energy per unit of surface calculated to be $7 \times 10^{-3}{\mathrm{~J} . \mathrm{m}^{-2} \text { ? }}^{\text {. }}$ This would lead to an energy of the two facing grain boundaries estimated around $6 \times 10^{-9} \mathrm{~J}_{\mathrm{m}} \mathrm{m}^{-1}$, to be compared with the energy of a greennematic 2D defect of length $L=460 \mathrm{~nm}$

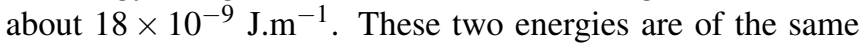
order of magnitude, suggesting an easy transition from one 
structure to the other one. This could occur in presence of an amount of nanoparticles large enough to efficiently stabilize the dislocations, due to their size of the same order than the dislocation core size. Moreover, the structure here revealed is expected to be the one of all $8 \mathrm{CB}$ films on substrates imposing a planar unidirectional anchoring together with an anchoring energy equal or higher than the one on rubbed PVA, associated with perpendicular layers covering all the substrate. As a comparison, the curve of the period as a function of the thickness is different on $\operatorname{MoS}_{2}\left(P=4.4 e+300^{*}\right)$ and leads to larger periods for the same thicknesses with respect to rubbed PVA substrates. It suggests for example another structure for the transition sublayer in relation with a smaller smectic zenithal anchoring energy on $\mathrm{MoS}_{2}$ with respect to rubbed PVA substrates. The corresponding transition sublayer could be shared in two parts : one part with grain boundaries joining parallel and perpendicular smectic layers, another part with a parallel smectic layer directly on the substrate. As a consequence one would expect a different density of dislocations on $\mathrm{MoS}_{2}$ with respect to rubbed PVA. The nature of the substrate may thus influence the nature of the array of dislocations in the oily streaks if the anchoring energy is not high enough.

\subsection{Conclusion}

Combining polarized optical microscopy, X-ray diffraction and ellipsometry, we have determined the internal structure of linear defect domains in thin films of smectic liquid crystals, confined between air and rubbed PVA, inducing homeotropic and planar unidirectional anchoring, respectively. The hybrid anchorings curve the smectic layers in flattened hemicylinders. We evidence that these distorted films can be viewed as a selective assembly of topological defects with various degree of disorder. They comprise an array of straight parallel edge dislocations of different Burger vectors and we determine their localization. A rotating grain boundary is created by removing curved layers close to the axis of curvature, where the curvature energy is maximum. This is in agreement with similar results obtained on crystalline substrates ? ?, thus demonstrating the generic character of this grain boundary. The shape of the rotating grain boundary reduces the number of dislocations, which are eliminated from the bottom of the boundary and concentrated at its summit. We evidence the presence of a transition sublayer connecting the curved and flat horizontal smectic layers to the substrate, allowing for a complete coverage of the PVA substrate with perpendicular smectic layers. This configuration minimizes the zenithal anchoring energy for the planar unidirectional geometry that is characterized by a very large anchoring strength, $W \geq 10^{-2} \mathrm{~J} . \mathrm{m}^{-2}$. A nematic $2 \mathrm{D}$ defect is required to join the flat parallel layers in the central region of the hemicylinders with the perpendicular layers of the transition sublayer. It may be melted in a nematic area, this melting being more favorable than the formation of dislocations within the transition sublayer. Dislocations of large burger vectors are thus localized at the summit of the rotating grain boundary, small Burger vector dislocations are located at the basis of the curvature walls between neighboring hemicylinders ${ }^{\text {? }}$ and nematic 2D defect between the transition sublayer and the central region of the hemicylinders . In a future work, the question of the evolution of smectic oily streaks in presence of nanoparticles should be addressed. The similarity between the energies of melted grain walls and grain boundaries with dislocations in the transition sublayer indeed suggests a possible structural evolution in presence of nanoparticles.

\subsection{Acknowledgement}

We thank Christophe Rafaillac and Benjamin Voisin for sample holder elaboration and technical support on SIXS beamline, respectively. We thank Frederic Picca for the extraction of experimental data. We finally acknowledge Charles Rosenblatt and Rolfe Petschek for useful discussions. This work was partly supported by the PNANO program of the French national research agency (ANR) through grant ANR-09-Nano003, Nanodiellipso, and by the Partner University Fund, administered by the Embassy of France in the United States. 